\title{
Notas sobre la concepción de modelo por los manuales de técnicas de investigación social
}

\author{
Javier Callejo
}

¿De qué se habla cuando se utiliza el concepto modelo? En principio, ha de reconocerse en el término una notable labilidad. Ello permite conducirlo a los más diversos contextos. Es una herramienta conceptual con la capacidad de quedar bien en casi todos los ámbitos. Tal vez aquí resida su éxito en las ciencias sociales (Ander-Egg 1990:83, Sjoberg y Nett 1980:43). Puede servir para un roto y para un descosido, algo especialmente pertinente hablando del significante modelo.

Sin embargo, su labilidad también trabaja en contra del concepto, haciéndolo confuso, enormemente escurridizo, como ocurre con casi todos los significantes polisémicos. En especial, cuando se quiere enseñar a los futuros profesionales de las ciencias sociales qué es un modelo y para qué sirve. Teniendo esto en cuenta, se hace un esquemático repaso de lo que se entiende por modelo en un instrumento tan vital, como discutido, en la enseñanza de la metodología de las ciencias sociales como los manuales de técnicas de investigación social.

Se toma como referencia un conjunto de manuales de técnicas de investigación social de amplio uso por los anteriores y actuales alumnos, ya que son referencia básica en los programas académicos existentes en la Universidad española, o lo han sido en un momento dado. No están todos los manuales de esta materia publicados en castellano; pero la selección se ha hecho bajo el criterio de relevancia, es decir, tomando los que se han considerado más relevantes en la pequeña historia institucional de la materia.

En este breve repaso, se podrá observar que el significado del concepto de modelo no es tan unidimensional como, en principio, cabía esperar tratándose de manuales. Son distintos sus significados y, sobre todo, varía de un manual a otro la relación que se establece entre modelo y teoría. 


\section{LA DIVERSIDAD DE SIGNIFICADOS}

Para la Real Academia de la Lengua, tras pasar las acepciones más relacionados con la moda y lo vestimentario, el término modelo presenta dos definiciones que pueden considerarse cercanas al campo metodológico:

- Representación en pequeño de alguna cosa.

- Esquema teórico, generalmente en forma matemática, de un sistema o de una realidad compleja (por ejemplo, la evolución económica de un país), que se elabora para facilitar su comprensión y el estudio de su comportamiento.

Hasta diez significados distintos ofrece de modelo el Diccionario de la Real Academia. Pero los ocho restantes quedan lejanos de nuestra materia. De hecho, el segundo parece el adecuado para ser tomado en cuenta y generalizarlo en la didáctica de la investigación social; mientras que el primero recoge la metáfora, tan usada, del modelo como maqueta, al igual que las utilizadas por los arquitectos y los ingenieros (Manheim y Rich 1988:256): el modelo como cuadro simplificado, reducido y manejable del mundo.

La mayor parte de los manuales subrayan la función señalada en la definición anterior: hacer comprensible ciertos fenómenos o comportamientos sociales complejos. Es decir, prácticamente todos, pues es difícil pensar en fenómenos sociales simples, sin complejidad.

Dentro de las diferencias que se verán más adelante, también hay coincidencias. Se puede hablar de asunción generalizada de que el modelo no es la realidad sino que sirve para explicar ésta. El modelo recoge algunas de las características esenciales del mundo; pero no puede recoger todas, pues, entonces, sería el mundo. Sería tan complejo como el mundo. Como el mapa borgiano, que es de escala igual o superior a la del territorio, tiene poca utilidad. No obstante, si bien en los manuales se acentúa la separación entre modelo de la realidad y la realidad como modelo, hemos de ser consciente de que tal frontera queda frecuentemente rota por la práctica de investigación y, sobre todo, por la práctica institucionalizada de investigación, pues la posesión de un modelo es lo que tiende a definir la categoría de sabio o científico.

Sin embargo, el acuerdo no parece tan extendido cuando se aborda la relación entre modelo y teoría, por un lado, y modelo y expresión matemática. Además, la primera definición deja a un lado de la concepción de modelo expresiones como «modelo estadístico», pues no es un esquema teórico que explique un comportamiento. Aparece como algo más general. Menos apegado a un caso específico. Apego que es subrayado por la definición de Seco, Andrés y Ramos (1999), cuando ponen por ejemplo de tales esquemas teóricos el «modelo sueco» o la falta de «modelos en la izquierda». Parece que no se está hablando de los mismo al utilizar el término modelo.

La sensación de que el término modelo tiene diversos significados no es nueva. En relación con los indicadores, Casas Aznar (1989) es consciente 
de la circulación de diversos significados atribuidos al término modelo. Por ello, destaca cuatro maneras diferentes de hablar de modelo:

1) Como prototipo o tipo ejemplar de algo a lo que habría que aspirar. Modelo como pattern, siguiendo la tipología de Ander-Egg (1990:84).

2) Una serie de planos esquemáticos, mostrando qué es una cosa o cómo hay que desarrollar una cosa. Esta concepción de modelo se encuentra precisamente extendida en los manuales encargados de describir las etapas del proceso de investigación, donde no falta una especial tendencia tan normativa, como ideal.

3) Un procedimiento de análisis de datos para propósitos generales, sin contenido, como cuando se habla, por ejemplo, del modelo subyacente en el modelo de regresión lineal o de cualquier otra técnica analítica. Desde tal perspectiva, toda técnica de análisis supone un modelo.

4) Un modelo matemático, como los especialmente utilizados por la econometría, como disciplina social que mayor uso ha hecho de la modelización.

Ahora bien, la que defiende Casas Aznar como modelo-modelo, como modelo al que hay que aspirar, es la cuarta acepción. Es decir, incrusta primera y cuarta acepciones. Es lo que le permite retomar la relación con la teoría, que no aparece manifiestamente en ninguno de los cuatro significados: «Un modelo en el que todos los conceptos han sido operacionalizados y las relaciones entre ellos han sido validadas es una teoría»(Casas 1989:125). El horizonte del modelo, tras pasar por las matemáticas (operacionalización), es la teoría. Relación entre teoría y modelo que no siempre quedará igualmente definida.

A las cuatro acepciones, pueden añadirse, al menos, otras tres:

5) Cercana a la 2, como articulación de teorías que explican un fenómeno (Sierra 1979, Álvaro y otros 1996), que revelan el «mecanismo oculto» de un fenómeno (Ander-Egg 1990).

6) Acción de hacer común lo que parece distante. Cuando se dice de varios fenómenos o comportamientos que siguen el mismo modelo, se está señalando lo que tienen de común (Manheim y Rich 1988), aun cuando sea relativamente abstracto. Se parece más a la concepción weberiana de tipo ideal, que a la de ejemplaridad -expuesta en el anterior punto 1 - en la medida que una construcción rigurosamente racional con arreglo a fines, pero irreal o vacía (Weber 1979:16), sirve para comprender una variedad de casos y acciones reales concretas influidos por irracionalidades.

7) Corriente de métodos actualmente utilizado, como se señala en el texto coordinado por Clemente (1992:56), que es una especie de metamanual en alguno de sus fragmentos (manual que habla de lo que dicen otros manuales), o como especial enfoque en el ámbito de una disciplina, como hacen Tomás Ibáñez y Lupicinio Iñíguez, al hablar de 
modelos de investigación en Psicología Social Aplicada desde una posición crítica, para referirse al enfoque cualitativo (Álvaro y otros 1996:68ss.). Se acerca esta concepción a la idea de paradigma, según la tipología de Ander-Egg (1990:84), aun cuando aquí nos encontramos con otro término que, desde Kuhn, ha sido usado de las más variadas maneras, hasta convertirlo en un término inútil, pues sirviendo para todo, no sirve para nada.

Ha de destacarse que varias de estas concepciones pueden encontrarse en el mismo manual, sin señalarse las diferencias. Algo que es más común en los manuales escritos por varios autores (König 1973, Álvaro 1996, Morales 1997).

\section{MODELO COMO RELACIÓN ENTRE VARIABLES QUE EXPLICAN}

Los modelos hablan de la realidad. Éste es su principal papel, ya lo hagan directa o indirectamente. Incluso, desde la Psicología Social, se destaca su capacidad de actuar sobre la realidad. Así, el modelo tiene su proyección en el trabajo de la memoria: «Estos modelos postulan que los nodos o conceptos que tengan más relaciones serán más fáciles de recordar....” (en J. F. Morales 1997:135). Aceptado un modelo, se utiliza en la práctica, como es el de establecer nodos con más relaciones, para que sean más recordados. Pero, en principio, la aplicación práctica del modelo aparece como finalidad de la investigación en general. El modelo quedaría, en principio, remitido a una explicación de la realidad en términos de relaciones entre variables, $y$, como objetivo ideal, a la posibilidad de predicción de fenómenos y comportamientos.

El modelo habla de la realidad; pero no lo hace de cualquier forma. El modelo desarrolla esquemáticamente las relaciones lógicas entre variables. ¿Hasta dónde llegan estas relaciones?

Como señalan Josep M. ${ }^{a}$ Blanch o Alicia Garrido, se habla del modelo de Jahoda, de Warr o de la agencia, para explicar las consecuencias del desempleo (Álvaro y otros 1996:100), de una realidad. El modelo es una relación entre variables que explican un fenómeno, la nostalgia del desempleo en este caso. Modelo como teoría o conjunto de teorías, he aquí un dilema momentáneamente menor, que explican de manera causal un fenómeno: «El modelo teórico de Beck explica la depresión como una consecuencia de experiencias vividas en la infancia...» (Álvaro y otros 1996:390). El modelo, con las flechas de su representación que indican algún tipo de condicionamiento o determinación, expresa una relación causal. Por lo tanto, no se trata de una relación cualquiera entre variables, sino que la idea de modelo implica relación causal entre las mismas. Es lo que aparece condensado en ese instrumento gráfico que es la punta de la flecha. De hecho y aun cuando se especifica como un modelo particular, el modelo causal (por ejemplo, Briones 1982), aparece como el modelo-modelo, moviéndonos nuevamente en el ámbito de 
lo deseable. Causalidad que las disciplinas sociales más próximas a la experimentación, como la psicología social de la que se obtienen las citas anteriores de Álvaro, Garrido y Torregrosa, asumen en mayor medida que otras disciplinas menos experimentales, como sociología, antropología, política o historia.

Siguiendo el mismo manual, se obtienen concepciones que ahondan en esta idea de la explicación causal, realizados por otros autores distintos, como es el caso de José Luis Álvaro y Darío Pérez: «el modelo presentado por Warr está centrado en el estudio de los determinantes del modelo social sobre la salud mental» (Álvaro y otros 1996:387). Es decir, el modelo está determinado (explicado) por otro modelo (el social): el modelo explica cómo la realidad es causada por otro modelo. Pero, en la misma página, se ofrece otra concepción de modelo, ya sin el peso de la causalidad. Estamos, entonces, ante simples modelos teóricos-descriptivos: «En el modelo teórico presentado por Mirosky y Ross, el deterioro en la salud mental se manifiesta como un estado subjetivo de malestar caracterizado por la depresion y la ansiedad y sus correlatos fisiológicos y emocionales» (Álvaro y otros 1996:387).

El argumento apuntado de un modelo que explica otro modelo, con la confusión terminológica que puede generar en estudiantes que se inician en esta lenguaje, se encuentra en otras obras. En la mayor parte de las ocasiones, tal relación entre modelos es un interesante ejercicio de reflexión y que, en definitiva, puede señalar que nuestra relación con los modelos va más allá de los instrumental. Se tiene la sensación de que la actividad de conocer o de investigar se encuentra en una telaraña de modelos, de manera que se observan o aplican (y verifican, siguiendo otra tradición) modelos desde modelos. Pero ha de reconocerse que este último argumento no se suele añadir a textos que utilizan diversas concepciones de modelo. La diversidad se halla incluso en el mismo párrafo, como ocurre en el siguiente fragmento: «El modelo más frecuente es el de $W$. Wallace. La actividad científica es descrita por Wallace como un proceso dinámico interactivo entre la realidad y los modelos o teorías que la explican» (González 1997:18), donde la autora articula el significado de modelo como algo ideal - "elaboración teórica desde una perspectiva ideal», siguiendo unas palabras que resaltan la idea de modelo como algo deseable-con el de conjunto de teorías explicativas. De hecho, resulta llamativo cómo es criticada la primera concepción de modelo, como algo ideal -«el modelo de Wallace padece los inconvenientes que son propios de todo modelo, como es el elevado grado de abstracción en lo que se refiere a las contingencias de la praxis científica» (González 1997:20)- de la que se ha de derivar implícitamente una crítica a la segunda, pues el primer significado de modelo parece de un nivel lógico superior al segundo o instrumental.

\section{LA RELACIÓN CON LA TEORÍA}

La relación entre teoría y modelo es fluctuante. Para unos, el modelo es un conjunto de teorías que explican un fenómeno (Sierra 1979, Álvaro y Páez 
1996). Para otros, la teoría es lo que subyace a un modelo; mientras que, en la posición contraria, se encuentran quienes mantienen que toda teoría arrastra un modelo (del hombre, de la sociedad, de las relaciones sociales, etc.). De esta manera, conjuntando las dos últimas relaciones, podría concluirse que un modelo (explicativo) lleva implícito un modelo de los objetos sociales. Por último, se encuentra la relación creativa entre modelo y teoría. Para éstos, el modelo es fuente creativa de teorías. Así lo entienden José Francisco Valencia, al señalar que: «el propio Ajzar ha reformulado el modelo, dando lugar al surgimiento de la teoría de la acción planificada» (en Morales 1997:617); Sierra Bravo, al afirmar que los modelos extienden el ámbito de aplicación de las teorías (Sierra 1979:43); o Visauta (1989:60), al definir el modelo como un sistema que da por resultado una teoría.

La fluctuación de significados entre los conceptos de modelo y teoría hace que ocasionalmente se utilicen de manera indistinta: Manheim y Rich (1988:260) lo hacen al hablar de teoría informal y modelo informal como sinónimos. La ambigüedad, rasgo inequívocamente rechazado en el perfil y la actuación del investigador, se apodera de esta relación: «Al revisar la literatura al respecto, nos encontramos con definiciones contradictorias o ambiguas, además, conceptos como 'teoría', 'orientación teórica', 'marco teórico de referencia', 'esquema teórico' o 'modelo' se usan ocasionalmente como sinónimos y otras veces sólo con leves matices diferenciales» (Hernández y otros 1991:38). Ha de destacarse que el manual que produce la última referencia, de Hernández, Fernández y Baptista, apenas desarrolla el concepto de modelo en algún ejemplo, tras señalar la situación de ambigüedad entre teoría y modelo. Sin intención de constituirse en conclusión, se destaca que los manuales que más hincapié hacen en la metodología experimental, como los de Hernández y otros (1991), León y Montero (1993) o el coordinado por Miguel Clemente (1992), tienden a dejar a un lado el concepto de modelo.

Siguiendo al economista Papandreou, Sjoberg y Nett (1980:43) distinguen entre teoría y modelo. La primera es un sistema lógico-deductivo que tiene la condición de falsabilidad. El segundo es un sistema conceptual que carece de tal condición, pero es capaz de confirmación empírica, lo cual no deja de ser un tanto paradójico. Más cuando algunas páginas después (pág. 287), queda definido el modelo como sistema deductivo, haciéndose especial énfasis en sus peligros.

Ander-Egg se enfrenta al problema de las similitudes y diferencias entre modelo y teoría de la mano de Kaplan: «con la teoría aprendemos algo acerca del asunto, pero no al investigar las propiedades de la teoría (como ocurre con un modelo). La teoría establece que el asunto tiene una cierta estructura, pero la teoría no exhibe necesariamente esa misma estructura (tal como hace un modelo)» (Ander-Egg 1990:86). Siguiendo esta afirmación, a la que no puede calificarse de transparente, el modelo señala la estructura del fenómeno social; pero no es teoría. La teoría queda relegada a decir algunas cosas, sin estructura, del objeto social.

El embrollo parece salvado, hasta que nos encontramos con una definición de teoría que, como era esperable entre quienes recuerden algo de sus 
estudios filosóficos, incluye el concepto de estructura: definiremos una teoría como un conjunto de hipótesis estructurado por la relación de implicación o deducibilidad o, más formalmente: una teoría $T$ es una estructura $(H, I)$ en la que $\underline{H}$ es un conjunto de hipótesis $\mathrm{e} I$ es una relación en $\underline{H}$ llamada «implicación» o "deducibilidad», de manera que $\underline{H}$ está débilmente conectado por I» (Galtung 1966:538). Si a esto se añade que Galtung presenta como dimensiones de las teorías: generalidad, amplitud, evaluación de las hipótesis, formalización, axiomatización, relación con otras teorías, predicibilidad, comunicabilidad, reproducibilidad y fecundidad; se vuelven a nublar las diferencias entre teoría y modelo. Algo parecido ocurre con el papel dado por Goode y Hatt (1967:18 ss.) a la teoría como: orientación, conceptualización y clasificación, resumen, predicción de los hechos e indicadora de los claros en nuestros conocimientos.

Al igual que Galtung o Goode y Hatt, Grawitz tampoco utiliza el término modelo. El espacio dejado por este concepto es ocupado por la caracterización de teoría. Así, habla de teorías matemáticas aplicadas a las ciencias sociales (Grawitz 1975:413), al señalar lo que hoy se denominan modelos matemáticos derivados de teoría de grafos, juegos, etc. Se constata una observación redundante: allí donde no se utiliza o queda al margen el concepto de modelo, tiende a ocupar su espacio el concepto de teoría. Sin embargo, en los manuales que se da relevancia al concepto de modelo, las dimensiones o papel de la teoría queda reducido.

La idea de estructura como elemento diferencial, apuntada por AnderEgg, ha de derivarse de la capacidad representativa del modelo. Aun cuando tal cuestión no es indicada por el autor, suena extraña la idea de teoría sin estructura. La relación entre teoría y modelo se encuentra en que, como indica Visauta, el modelo: «representa a la teoría» (Visauta 1989:59).

La estructura está en la representación, siendo ésta la aportación del modelo frente a lo que serían simples teorías. A través de la representación. se relacionan teorías. Así, lo que viene a representar un modelo no es la realidad, entendida como relación entre variables de la realidad, sino la relación del conjunto de teorías operantes. Esto es lo que parece indicar González Río: «Un modelo expresa las relaciones entre elementos que son percibidas por la teoría, siendo más una reproducción teórica de la realidad que una reproducción de ésta» (González 1997:20). El modelo introduce otro lenguaje, que se añade al de la teoría. Pero resulta curioso comprobar cómo tal significado apenas se encuentra directamente expresado en los manuales que abordan la cuestión. En la medida que se habla de esquema, parece indicado en la propia propuesta de la Real Academia: como esquema teórico, explicativo de la realidad. El modelo añade el esquema a la teoría, pone en forma a ésta.

Ante la confusión, ninguno de los autores que abordan directamente la cuestión, en uno de los manuales que por distintos motivos cabe considerar clásico, ven diferencia entre teoría y modelo. Para Hans Albert: «el modelo tiene el status lógico de una teoría empírica y no se diferencia, por consiguiente, de las teorías cientificas» (en König 1973:83). Puede hablarse, a lo sumo, del modelo como de una especificación de la teoría: la empírica o científica. Para 
Hans L. Zetterberg (en König 1973:92 y 93), modelo y teoría son el mismo resultado de la actividad teórica. El matiz es puesto, en el segundo autor, en el cariz hipotético y explicativo del modelo, por un lado, y, sobre todo, en la intrínseca capacidad de los modelos para traducir la teoría a lenguajes distintos del natural, como el simbólico o el matemático. Es decir, teoría y modelo serían lo mismo; pero con distintas capacidades expresivas, de manera que tienden a coincidir cuando el modelo es incapaz de representación en lenguaje distinto al natural.

\section{EL MODELO MATEMÁTICO EN EL HORIZONTE}

Se matiza que no todo modelo ha de proyectarse en una expresión matemática; pero ésta tiende a aparecer como la penúltima finalidad, antes de la predicción. No obstante su carácter matemático también está en el inicio: «en el sentido originario del término, utilizado en ciencia económica, un "modelo» presenta dos caracteristicas: en primer lugar, se trata de una construcción matemática y, en segundo lugar, de un instrumento de previsión" (Duverger 1962:381).

El acento en la explicación causal conduce a la predicción, manteniendo la sensibilidad determinista-mecanicista. El que se hace en la relación entre variables, a la formalización matemática. Si el modelo es representación teórica, ésta parece alcanzar el mayor grado cuando viene dada en caracteres matemáticos, cuando a la representación gráfica se añaden ecuaciones $o$, con mayor precisión, sistemas de ecuaciones. Es más, la predicción parece difícil sin la previa mediación de instrumentos de medida y su consecuente relación algorítmica.

Hay modelos y modelos matemáticos; pero los últimos son los verdaderos modelos, Si no se alcanza la expresión matemática, el modelo aparece como una explicación de segundo grado de la realidad. Así, como mantienen Manheim y Rich (1988:392 ss.), todo modelo, entendido principalmente como relación entre variables, ha de tener una representación matemática, ya sea en una o varias ecuaciones. De lo contrario, se queda en lo «informal».

Es tal la diferencia cualitativa entre modelos no matemáticos, que aparecen casi como cuasimodelos, y modelos matemáticos, que a Boudon (1981) le es más fácil separar entre uno y otro tipo de modelos que entre disciplinas como sociología, psicología, economía, antropología o historia. Tanto discurso sobre la especificidad del objeto de cada una de las disciplinas queda diluido en la radical separación entre un tipo de modelos y otros. Una tipología que no oculta su carácter de jerarquización entre un elemento marcado negativamente, el modelo no matemático, y lo que se considera como modelo pleno.

La disputa entre modelos puede llegar a centrarse en qué expresiones matemáticas se utilizan, como ocurre -siguiendo a Morales, Rebolloso y Moya (1997)- entre los modelos combinatorios del cambio de actitudes: ¿es más adecuada la suma o el promedio para representar un conjunto de actitudes? Según sea la respuesta, nos encontraremos en un modelo u otro. 
No todos los autores sitúan acríticamente las matemáticas como punto de llegada de la modelización. También es frecuente el escepticismo (Duverger 1962:382). A este respecto destaca la posición de Zetterberg, por ser alguien poco dudoso de antimatematicismo: «No existe ninguna duda de que las matemáticas confieren a la construcción de modelos una precisión mayor. Sin embargo, el grado de precisión requerido para un modelo debe ser determinado, de una parte, por la calidad del material a comprobar y, de otra, por la posible utilidad práctica del modelo. La precisión por sí misma es una virtud dudosa y aburrida» (en König 1973:119). Un precioso consejo para investigadores ideales $y$, sobre todo, una advertencia para quienes, una vez establecido el modelo matemático, van de la realidad al modelo sin pasar por la reflexión de su adecuación al objeto estudiado: «Sucede aun que, al jugar a la competencia matemática como otros juegan a una cultura literaria o artística, parece buscar desesperadamente el objeto concreto al que tal o cual modelo formal se puede aplicar. Sin duda los modelos de simulación pueden tener una función heurística, al permitir imaginar modos de funcionamiento posibles. Pero aquellos que los construyen se abandonan a menudo a la tentación dogmática que Kant denunciaba ya en los matemáticos y que lleva a pasar del modelo de la realidad a la realidad del modelo» (Bourdieu 1988:56, también en 1991:70). Con ello, nos salimos momentáneamente de los manuales, aun cuando está en ellos. Como cuando se define un modelo como una: «estructura a la que debe adaptarse un fenómeno social, según una serie de probabilidades que se conocen previamente por la experiencia» (Vázquez y López 1962: 402), en uno de los manuales que tiene la «transparente inocencia» de ser de los primeros que obtuvo una importante circulación en las aulas. Ya no es el modelo el que intenta capturar un fenómeno social, sino que es éste el que ha de integrarse en el modelo.

\section{COMENTARIOS FINALES}

No todo lo relativo a la investigación social se aprende en los manuales; pero parece conveniente que hubiese cierta comunidad de conceptos en sus textos, pues es lo que, en definitiva, hace la comunidad profesional y científica. Es decir, de cara a éstas, es interesante que todos hablasen de la misma cosa cuando utilizan el término modelo. Como no parece posible una especie de «gran acuerdo», pues desde hace más de treinta años se viene constatando los múltiples y variados significados del concepto, se invoca cierto rigor, de manera que se diga lo que se quiere decir cuando se utiliza el término. Una utilización del término que tiende a ocupar un lugar menos relevante, según se van sucediendo los manuales. Puede decirse que ocupa un lugar relativamente central en los más «antiguos», como los de König (1973), Sierra (1979), Manheim y Rich (1988) o Ander-Egg (1990, con numerosas ediciones previas), cuando: «desde hace algunos años, el término «modelo» es muy empleado en las ciencias sociales. Incluso llega a decirse que forma parte del vocabulario de moda» (Duverger 1962), lo que se proyecta en los manuales 
originariamente escritos entre finales de los cincuenta y el decenio siguiente. Sin embargo, es marginal en los de creación más reciente, como los de García Ferrando, Ibáñez y Alvira (1990) o Hernández, Fernández y Baptista (1991); o se inclina hacia un significado distinto, cercano al de paradigma o corriente en una disciplina (Álvaro, Garrido y Torregrosa 1996; Morales 1997).

Por otro lado, ha de tomarse una posición y afirmar que algunas de sus acepciones parecen menos acertadas que otras. Así, hablar de «modelo estadístico», al referirse al conjunto de reglas depositadas en la disciplina estadística cuando se aplica a las ciencias sociales, parece fuera de lugar. Pues, entre otras cosas, no hay un sólo modelo en la estadística. Como, por otro lado, tampoco puede hablarse de un «modelo cualitativo». Lo que fundamentalmente introduce la estadística es que la relación entre las variables, que es lo que representan los modelos, no se establece en términos deterministas sino probabilísticos.

Se ha destacado que una de las partes menos transparentes, a la hora de abordar el concepto de modelo por los manuales, se encuentra en su relación con la teoría. Con la salvedad que implica tratarse de un acercamiento a través de lo que los propios manuales dicen, parece que la diferencia fundamental no es tanto sustancial, como formal, de ejercicio de formalización y capacidad de los modelos para expresar la teoría en otro lenguaje, lo que, paradójicamente, supone un cambio sustancial. La aportación fundamental del modelo consiste en resumir en una figura coherente todo el trabajo teórico anterior. Un punto y aparte en la actividad teórica que, como resaltan algunos de los manuales abordados, posibilita la creación de nuevas teorías.

Poner la teoría en un plano, primero, y en ecuaciones, después, supone un esfuerzo de formalización. Pertinente e incluso necesario para explicar algunos fenómenos. Pero también es un esfuerzo de sincronización, de establecimiento de las relaciones fuera del tiempo, para controlar su coherencia. Una exclusión del tiempo que la observación de las relaciones sociales admite sólo de vez en cuando y con justificados matices. Una exclusión siempre ideológica, siendo uno de los aspectos que primeramente se mutilan de la realidad, siguiendo el término traducido de Bourdieu: «Los modelos formales nunca revelan tan completamente la que es probablemente su más indiscutible virtud, es decir, su poder de revelar a contrario la complejidad de lo real que mutilan» (Bourdieu 1991:83).

\section{BIBLIOGRAFÍA}

Álvaro, J.L., A. Garrido y J.R. Torregrosa (coord.) (1996): Psicología Social Aplicada, Madrid: McGraw-Hill.

ANDER-EGG, E. (1990): Técnicas de investigación social, México: Ateneo.

Boudon, R. (1981): «Modelos y métodos matemáticos» en VV.AA., Corrientes de la investigación en las ciencias sociales, Madrid: Tecnos-Unesco.

Bourdieu, P. (1988): Cosas dichas, Barcelona: Gedisa. 
Bourdieu, P. (1991): El sentido práctico, Madrid: Taurus.

Brones, G. (1982): Métodos y técnicas de investigación para las ciencias sociales, México: Trillas.

Casas AZNAR, F. (1989): Técnicas de investigación social: los indicadores sociales y psicosociales, Barcelona: PPU.

Clemente Diaz, M. (coord.) (1992): Psicología Social. Métodos y Técnicas de Investigación Social, Madrid: Eudema.

Duverger, M. (1962): Métodos de las Ciencias Sociales, Barcelona: Ariel.

Galtung, J. (1966): Teoría y Método de la Investigación Social, Buenos Aires: Eudeba.

García Ferrando, M., J. Ibáñez y F. Alvira (1990): El análisis de la realidad social. Madrid: Alianza.

González Ró, M. J. (1997): Metodología de la investigación social. Técnicas de recolección de datos, Alicante: Aguaclara.

Goode, W.J. y P.K. Hatt (1967): Métodos de investigación social, México: Trillas.

Grawrrz, M. (1975): Métodos y técnicas de las ciencias sociales, Barcelona: Hispano Europea.

Hernández Sampieri, R., C. Fernández Collado y P. Baptista lucio (1991): Metodología de la investigación. México: McGraw-Hill.

Könıl, R. (1973): Tratado de sociología empírica, Madrid: Tecnos.

LEÓN, O.G. e I. Montero (1993): Diseño de investigaciones. Introducción a la lógica de la investigación e Psicología y Educación. Madrid: McGraw-Hill.

Manheim. J. B. y R. C. Rich (1988): Análisis político empírico. Métodos de investigación en ciencia política, Madrid: Alianza.

Morales, J. F. y M. C. Moya (1997): Psicología Social, Madrid: McGraw-Hill.

Morales, J. F. y otros (1997): Metodología de la psicología. Madrid: UNED.

Seco, M., O. Andrés y G. Ramos (1999): Diccionario del español actual, Madrid: Aguilar.

Sierra Bravo, R. (1979): Técnicas de Investigación Social. Madrid: Paraninfo.

SjoBerg, G. y R. Netr (1980). Metodología de la investigación social, México: Trillas.

VÁzquez, J.M. y P. López Rivas (1962): La investigación social, Madrid, O.P.E.

Visauta, B. (1989): Técnicas de Investigación Social, Barcelona: P.P.U.

Weber, M. (1979): Economía y Sociedad, México: Fondo de Cultura Económica (e.o. 1922). 